\title{
Error Estimates for Solutions of the Semilinear Parabolic Equation in Whole Space
}

\author{
Xiaomei $\mathrm{Hu}^{1,2}$ \\ ${ }^{1}$ School of Mathematics and Statistics, Hubei University of Science and Technology, Xianning 437100, China \\ ${ }^{2}$ School of Mathematics and Statistics, Central China Normal University, Wuhan 430079, China
}

Correspondence should be addressed to Xiaomei Hu; xiaomeihu@163.com

Received 1 June 2014; Accepted 28 June 2014; Published 8 July 2014

Academic Editor: Caidi Zhao

Copyright (c) 2014 Xiaomei Hu. This is an open access article distributed under the Creative Commons Attribution License, which permits unrestricted use, distribution, and reproduction in any medium, provided the original work is properly cited.

\begin{abstract}
This paper is focused on the error estimates for solutions of the three-dimensional semilinear parabolic equation with initial data $u_{0} \in L^{2}\left(\mathbb{R}^{3}\right)$. Employing the energy methods and Fourier analysis technique, it is proved that the error between the solution of the semilinear parabolic equation and that of linear heat equation has the behavior as $O\left((1+t)^{-3 / 8}\right)$.
\end{abstract}

\section{Introduction}

In this study we consider the Cauchy problem of the following three-dimensional semilinear parabolic equation:

$$
\begin{gathered}
\partial_{t} u-\Delta u+|u|^{p-2} u=0, \\
u(x, 0)=u_{0} .
\end{gathered}
$$

Here $p>5 . u(x, t)$ is the unknown function at the point $(x, t) \in \mathbb{R}^{3} \times(0, \infty)$ and $u_{0}$ is the initial data.

As an important partial differential equation, the wellposedness and asymptotic behavior of solutions of semilinear parabolic equation has attracted more and more attention and many important results have been investigated (see [1-4] and references therein). The mathematical model (1) can be seen as the heat equation with damping and friction effects. From the view on the mathematics point, the nonlinear damping $|u|^{p-2} u$ in (1) may increase the regularity of the weak solutions. However, it will be the main obstacle on the asymptotic behavior of the solutions to the semilinear parabolic equation (1). For the $n$-dimensional linear heat equation

$$
\begin{gathered}
\partial_{t} u-\Delta u=0, \\
u(x, 0)=u_{0} .
\end{gathered}
$$

The fundamental solution is

$$
e^{t \Delta}=E(x-y, t)=(4 \pi t)^{-n / 2} e^{-|x-y|^{2} / 4 t},
$$

and the solution of (2) is expressed as

$$
\begin{aligned}
u(x, t) & =e^{t \Delta} u_{0}=\int_{\mathbb{R}^{n}} E(x-y, t) u_{0}(y) d y \\
& =(4 \pi t)^{-n / 2} \int_{\mathbb{R}^{n}} e^{-|x-y|^{2} / 4 t} u_{0}(y) d y .
\end{aligned}
$$

In particular, the solution $u(x, t)$ of the linear heat equation

(2) exhibits the following asymptotic behavior (see [5]):

$$
\left\|e^{t \Delta} u_{0}\right\|_{L^{q}\left(\mathbb{R}^{n}\right)} \leq C t^{-(n / 2)(1 / p-1 / q)}\left\|u_{0}\right\|_{L^{p}\left(\mathbb{R}^{n}\right)}, \quad t>0
$$

Compared with the behavior of heat equation (2), it is an interesting problem to consider the influence of the linear damping $|u|^{p-2} u$ in the semilinear parabolic equation (1).

Motivated by the asymptotic results on some nonlinear differential equations in [6-9], in this study we will investigate the asymptotic error estimates between the solutions of both the semilinear parabolic equation (1) and the linear parabolic equation (2). Let us give an outline analysis of this question. On one hand, taking $p=q=2$ in (5), we only derive the bounds of the solution; that is,

$$
\left\|e^{t \Delta} u_{0}\right\|_{L^{2}\left(\mathbb{R}^{n}\right)} \leq C\left\|u_{0}\right\|_{L^{2}\left(\mathbb{R}^{n}\right)}, \quad t>0 .
$$


On the other hand, from the definition of weak solution for the semilinear parabolic equation (1) (see the definition in the next section), we also only get the $L^{2}$ bounds of weak solution for the semilinear parabolic equation (1) as

$$
\|u(t)\|_{L^{2}\left(\mathbb{R}^{n}\right)} \leq C\left\|u_{0}\right\|_{L^{2}\left(\mathbb{R}^{n}\right)}, \quad t>0 .
$$

By the direct computation, we only get the $L^{2}$ bounds of the error $u-e^{t \Delta} u_{0}$. It is obviously important to explore the explicit error estimates as time tends to infinity. In order to come over the main difficulty raised by the nonlinear damping $|u|^{p-2} u$, we will make full use of the Fourier analysis technique to explore the lower frequency effect of the nonlinear damping $|u|^{p-2} u$. Fortunately, we can control the nonlinear term $|u|^{p-2} u$ as

$$
\begin{aligned}
& \left(\int_{0}^{t}\|u\|_{L^{p-1}}^{p-1} d s\right)^{1 /(p-1)} \\
& \quad \leq\left(\int_{0}^{\infty}\|u\|_{10 / 3}^{10 / 3} d s\right)^{3 \theta / 10}\left(\int_{0}^{\infty}\|u\|_{p}^{p} d s\right)^{(1-\theta) / p} \leq C
\end{aligned}
$$

with

$$
\frac{1}{p-1}=\frac{\theta}{10 / 3}+\frac{1-\theta}{p} .
$$

This observation allows us to derive the explicit error estimates.

The remainder of this paper is organized as follows. In Section 2, we first recall some fundamental preliminaries and state our main results. In Section 3, we investigate the explicit error estimates of solutions between semilinear parabolic equation (1) and linear heat equation (2).

\section{Preliminaries and Main Results}

In this paper, we denote by $C$ a generic positive constant which may vary from line to line.

Let $\delta\left(\mathbb{R}^{3}\right)$ be the Schwartz class of rapidly decreasing functions (refer to [10]) and given $g \in \mathcal{S}\left(\mathbb{R}^{3}\right)$, its Fourier transformation $\mathscr{F} g$ or $\widehat{g}$ is defined by

$$
\mathscr{F} g(\xi)=\widehat{g}(\xi)=\int_{\mathbb{R}^{3}} e^{-i x \cdot \xi} g(x) d x
$$

$L^{p}\left(\mathbb{R}^{3}\right)$ with $1 \leq p \leq \infty$ denotes the usual Lebesgue space of all $L^{p}$ integral functions associated with the norm

$$
\|g\|_{L^{p}}= \begin{cases}\left(\int_{\mathbb{R}^{3}}|g(x)|^{p} d x\right)^{1 / p}, & 1 \leq p<\infty, \\ \operatorname{ess} \sup _{x \in \mathbb{R}^{3}}|g(x)|, & p=\infty .\end{cases}
$$
with

$H^{s}\left(\mathbb{R}^{3}\right)$ with $s \in \mathbb{R}$ denotes the fractional Sobolev space

$$
\|g\|_{H^{s}}=\left(\int_{\mathbb{R}^{3}}|\xi|^{2 s}|\hat{g}|^{2} d \xi\right)^{1 / 2} .
$$

To state our main results, let us firstly recall the definition of the weak solutions of the semilinear parabolic equation (1) (refer to [11]).
Definition 1. Given $u_{0} \in L^{2}\left(\mathbb{R}^{3}\right)$, a measurable function $u(x, t)$ on $\mathbb{R}^{3} \times(0, T)$ is called a weak solution to the semilinear parabolic equation (1) provided that

(1) $u \in L^{\infty}\left(0, T ; L^{2}\left(\mathbb{R}^{3}\right)\right) \cap L^{2}\left(0, T ; H^{1}\left(\mathbb{R}^{3}\right)\right)$, and $u \in$ $L^{p}\left(0, T ; L^{p}\left(\mathbb{R}^{3}\right)\right) ;$

(2) for any $\phi \in C_{0}^{\infty}\left(\mathbb{R}^{3} \times[0, T)\right)$

$$
\begin{aligned}
& \int_{0}^{T} \int_{\mathbb{R}^{3}}\left(u \cdot \partial_{t} \phi-\nabla u \cdot \nabla \phi-|u|^{p-2} u \phi\right) d x d t \\
& \quad=-\int_{\mathbb{R}^{3}} u_{0} \phi(0) d x ;
\end{aligned}
$$

(3) $u(x, t)$ also satisfies energy inequality

$$
\frac{1}{2} \frac{d}{d t} \int_{\mathbb{R}^{3}}|u|^{2} d x+\int_{\mathbb{R}^{3}}|\nabla u|^{2} d x+\int_{\mathbb{R}^{3}}|u|^{p} d x \leq 0 .
$$

Now our results read as follows.

Theorem 2. Suppose $u_{0} \in L^{2}\left(\mathbb{R}^{3}\right)$ and $u(x, t)$ is a weak solution of the Cauchy problem of the semilinear parabolic equation (1); one has

$$
\int_{\mathbb{R}^{3}}|u(t)-\tilde{u}(t)|^{2} d x=O\left((1+t)^{-3 / 4}\right) \quad \text { as } t \longrightarrow \infty,
$$

where $\widetilde{u}(t)$ is the weak solution of the linear heat equation; namely,

$$
\begin{gathered}
\partial_{t} \tilde{u}-\Delta \tilde{u}=0, \\
\tilde{u}(x, 0)=u_{0},
\end{gathered}
$$

with the same initial date $u_{0}$.

Remark 3. The result above seems inspiring. Since according to the $L^{p}-L^{q}$ estimates of the linear heat equation (16), we have only the $L^{2}$ bounds of the solution of linear equation (5); that is,

$$
\|\widetilde{u}(t)\|_{L^{2}\left(\mathbb{R}^{n}\right)} \leq C\left\|u_{0}\right\|_{L^{2}\left(\mathbb{R}^{n}\right)}, \quad t>0
$$

No asymptotic behavior of solution of linear equation (5) can be derived. Compared with the previous results on the time decay of the nonlinear partial differential equations models [12-15] where the initial data satisfies some additional conditions such as $L^{1}\left(\mathbb{R}^{3}\right)$, at the same time, for the nonlinear parabolic equation (1) with the same initial date $u_{0}$, the nonlinear damping term $|u|^{p-2} u$ is obviously not helpful for the asymptotic behavior of the semilinear parabolic equation (1). Therefore, it seems impossible to derive the asymptotic behavior of the difference between the semilinear parabolic equation (1) and the linear heat equation (16). Fortunately, we find a new trick which is different to the $L^{p}-L^{q}$ estimates to deal with the nonlinear term. This trick is mainly based on the Fourier analysis which allows us to explore successfully the lower frequency of the nonlinear damping term $|u|^{p-2} u$. 


\section{Error Estimates}

We are now in a position to investigate the explicit error estimate in this section. It should be mentioned that the global existence of the nonlinear parabolic equation can be proved by the standard contraction mapping principle (refer to [16]). Hence we only prove the error estimates. To carry out this issue, we develop some new tricks which mainly borrowed the idea in [17-20]. Denote the difference $w(t)=$ $u(t)-\widetilde{u}(t)$, where $u(t)$ and $\widetilde{u}(t)$ are the solutions of the semilinear parabolic equation (1) and the linear heat equation (16), respectively. Thus $w(t)$ satisfies the following system:

$$
w_{t}-\Delta w+|u|^{p} u=0, \quad w(x, 0)=0,
$$

in the weak sense. It is worth noting that the following derivation should be stated rigorously for the smooth approximated solutions and then take the limits to get the results of the weak solution of the semilinear parabolic equation (18). For convenience, we directly discuss weak solutions.

Multiplying both sides of (18) with $w$ and integrating in $\mathbb{R}^{3}$, it follows that

$$
\frac{d}{d t} \int_{\mathbb{R}^{3}}|w(t)|^{2} d x+2 \int_{\mathbb{R}^{3}}|\nabla w|^{2} d x=-2 \int_{\mathbb{R}^{3}}|u|^{p-2} u w d x,
$$

since

$$
\begin{aligned}
& -2 \int_{\mathbb{R}^{3}}|u|^{p-2} u w d x \\
& =-2 \int_{\mathbb{R}^{3}}|u|^{p-2} u(u-\tilde{u}) d x \\
& =-2 \int_{\mathbb{R}^{3}}|u|^{p} d x+2 \int_{\mathbb{R}^{3}}|u|^{p-2} u \tilde{u} d x \\
& \leq 2 \int_{\mathbb{R}^{3}}|u|^{p-2} u \tilde{u} d x, \\
& 2 \int_{\mathbb{R}^{3}}|u|^{p-2} u \tilde{u} d x \\
& \leq C\left(\int_{\mathbb{R}^{3}}|u|^{p-1} d x\right)\|\widetilde{u}\|_{L^{\infty}} \\
& \leq C\left(\int_{\mathbb{R}^{3}}|u|^{p-1} d x\right)(1+t)^{-3 / 4},
\end{aligned}
$$

where we have used the Hölder inequality and the $L^{p}-L^{q}$ estimates (5). Thus inserting the above inequalities into (19), one shows that

$$
\begin{aligned}
& \frac{d}{d t} \int_{\mathbb{R}^{3}}|w(t)|^{2} d x+2 \int_{\mathbb{R}^{3}}|\nabla w|^{2} d x \\
& \quad \leq C\left(\int_{\mathbb{R}^{3}}|u|^{p-1} d x\right)(1+t)^{-3 / 4} .
\end{aligned}
$$

Taking the Parseval inequality into consideration, it follows that

$$
\begin{aligned}
& \frac{d}{d t} \int_{\mathbb{R}^{3}}|\widehat{w}(\xi, t)|^{2} d \xi+2 \int_{\mathbb{R}^{3}}|\xi|^{2}|\widehat{w}(\xi, t)|^{2} d \xi \\
& \quad \leq C\left(\int_{\mathbb{R}^{3}}|u|^{p-1} d x\right)(1+t)^{-3 / 4} .
\end{aligned}
$$

Now multiplying both sides of (22) by $(1+t)^{3}$ together with direct computation, then we have

$$
\begin{aligned}
& \frac{d}{d t}\left((1+t)^{3} \int_{\mathbb{R}^{3}}|\widehat{w}(\xi, t)|^{2} d \xi\right) \\
& \quad+2(1+t)^{3} \int_{\mathbb{R}^{3}}|\xi|^{2}|\widehat{w}(\xi, t)|^{2} d \xi \\
& \leq C(1+t)^{2} \int_{\mathbb{R}^{3}}|\widehat{w}(\xi, t)|^{2} d \xi \\
& \quad+C\left(\int_{\mathbb{R}^{3}}|u|^{p-1} d x\right)(1+t)^{9 / 4} .
\end{aligned}
$$

Let

$$
S(t)=\left\{\xi \in \mathbb{R}^{3}:|\xi| \leq\left(\frac{3}{1+t}\right)^{1 / 2}\right\}
$$

then

$$
\begin{aligned}
(1+t)^{3} \int_{\mathbb{R}^{3}}|\xi|^{2}|\widehat{w}(\xi, t)|^{2} d \xi \\
=(1+t)^{3} \int_{S(t)^{c}}|\xi|^{2}|\widehat{w}(\xi, t)|^{2} d \xi \\
\quad+(1+t)^{3} \int_{S(t)}|\xi|^{2}|\widehat{w}(\xi, t)|^{2} d \xi \\
\geq(1+t)^{3} \int_{S(t)^{c}}|\xi|^{2}|\widehat{w}(\xi, t)|^{2} d \xi \\
\geq 3(1+t)^{2} \int_{\mathbb{R}^{3}}|\widehat{w}(\xi, t)|^{2} d \xi-3(1+t)^{2} \int_{S(t)}|\widehat{w}(\xi, t)|^{2} d \xi .
\end{aligned}
$$

Therefore

$$
\begin{aligned}
& \frac{d}{d t}\left((1+t)^{3} \int_{\mathbb{R}^{3}}|\widehat{w}(\xi, t)|^{2} d \xi\right) \\
& \leq(1+t)^{2} \int_{S(t)}|\widehat{w}(\xi, t)|^{2} d \xi \\
& \quad+C\left(\int_{\mathbb{R}^{3}}|u|^{p-1} d x\right)(1+t)^{9 / 4}
\end{aligned}
$$


then integrating in time, one shows that

$$
\begin{aligned}
(1+t)^{3} \int_{\mathbb{R}^{3}}|\widehat{w}(\xi, t)|^{2} d \xi \\
\leq C \int_{0}^{t}(1+s)^{2} \int_{S(s)}|\widehat{w}(\xi, s)|^{2} d \xi d s \\
\quad+C \int_{0}^{t}(1+s)^{9 / 4}\left(\int_{\mathbb{R}^{3}}|u|^{p-1} d x\right) d s \\
\leq C \int_{0}^{t}(1+s)^{2} \int_{S(s)}|\widehat{w}(\xi, s)|^{2} d \xi d s \\
\quad+C(1+t)^{9 / 4} \int_{0}^{t}\left(\int_{\mathbb{R}^{3}}|u(\tau)|^{p-1} d x\right) d \tau .
\end{aligned}
$$

In order to estimate the first term on the right hand side of (27), we take Fourier transformation to (18)

$$
\widehat{w}_{t}+|\xi|^{2} \widehat{w}=-\widehat{|u|^{p-2} u}, \quad \widehat{w}(0)=0
$$

the solution of the above ordinary differential equation is written as

$$
\begin{aligned}
|\widehat{w}(\xi, t)| & =\left|\int_{0}^{t} e^{-|\xi|^{2} t} \widehat{|u|^{p-2}} u d s\right| \\
& \leq C \int_{0}^{t}\left|\widehat{|u|^{p-2} u}\right| d s \\
& \leq C \int_{0}^{t}\left(\int_{\mathbb{R}^{3}}|u|^{p-1} d x\right) d s .
\end{aligned}
$$

On one hand, since $u$ is a weak solution of the semilinear parabolic equation (1) and according to the definition of weak solution and interpolation inequality, then for

$$
\frac{2}{r}+\frac{3}{q}=\frac{3}{2}, \quad 2 \leq q \leq 6 ;
$$

we have

$$
\begin{aligned}
& \left\{\int_{0}^{\infty}\left(\int_{\mathbb{R}^{3}}|u|^{q} d x\right)^{r / q} d t\right\}^{1 / r} \\
& \leq C \operatorname{ess} \sup _{0<t<\infty}\left(\int_{\mathbb{R}^{3}}|u|^{2} d x\right) \\
& \quad+C\left\{\int_{0}^{\infty} \int_{\mathbb{R}^{3}}|\nabla u|^{2} d x d t\right\}^{1 / 2} \leq C
\end{aligned}
$$

In particular, let

$$
r=q=\frac{10}{3}
$$

that is,

$$
\left\{\int_{0}^{\infty} \int_{\mathbb{R}^{3}}|u|^{10 / 3} d x d t\right\}^{3 / 10} \leq C .
$$

On the other hand, since $p>5$, that is,

$$
\frac{10}{3}<p-1<p
$$

hence applying the interpolation inequality,

$$
\begin{aligned}
& \left(\int_{0}^{t}\|u\|_{L^{p-1}}^{p-1} d s\right)^{1 /(p-1)} \\
& \quad \leq\left(\int_{0}^{\infty}\|u\|_{L^{10 / 3}}^{10 / 3} d s\right)^{3 \theta / 10}\left(\int_{0}^{\infty}\|u\|_{L^{p}}^{p} d s\right)^{(1-\theta) / p} \\
& \quad \leq C
\end{aligned}
$$

with

$$
\frac{1}{p-1}=\frac{\theta}{10 / 3}+\frac{1-\theta}{p} .
$$

Plugging the above estimates into (29), one shows that

$$
|\widehat{w}(\xi, t)| \leq C .
$$

Hence

$$
\begin{aligned}
& (1+t)^{3} \int_{\mathbb{R}^{3}}|\widehat{w}(\xi, t)|^{2} d \xi \\
& \leq C \int_{0}^{t}(1+s)^{2} \int_{S(s)} 1 d \xi d s+C(1+t)^{9 / 4} \\
& \quad \leq C \int_{0}^{t}(1+s)^{2}(1+s)^{-3 / 2} d s+C(1+t)^{9 / 4} \\
& \quad \leq C(1+t)^{3 / 2} d s+C(1+t)^{9 / 4} \\
& \quad \leq C(1+t)^{9 / 4} .
\end{aligned}
$$

That is,

$$
\int_{\mathbb{R}^{3}}|w(t)|^{2} d x=O\left((1+t)^{-3 / 4}\right), \quad t \longrightarrow \infty,
$$

which completes the proof of Theorem 2 .

\section{Conflict of Interests}

The author declares that there is no conflict of interests regarding the publication of this paper.

\section{Acknowledgment}

This work is partially supported by the NNSF of China with no. 11271148

\section{References}

[1] L. C. Evans, Partial Differential Equations, American Mathematical Society, Providence, RI, USA, 1998.

[2] A. Gmira and L. Veron, "Large time behaviour of the solutions of a semilinear parabolic equation in $\mathbb{R}^{N}$," Journal of Differential Equations, vol. 53, no. 2, pp. 258-276, 1984. 
[3] L. H. Zhang, "Sharp rate of decay of solutions to 2-dimensional Navier-Stokes equations," Communications in Partial Differential Equations, vol. 20, no. 1-2, pp. 119-127, 1995.

[4] C. Zhao, Y. Liang, and M. Zhao, "Upper and lower bounds of time decay rate of solutions to a class of incompressible third grade fluid equations," Nonlinear Analysis. Real World Applications, vol. 15, pp. 229-238, 2014.

[5] A. Pazy, Semigroups of Linear Operators and Applications to Partial Differential Equations, Springer, 1983.

[6] B. Dong and Y. Li, "Large time behavior to the system of incompressible non-Newtonian fluids in $\mathbb{R}^{3}$," Journal of Mathematical Analysis and Applications, vol. 298, no. 2, pp. 667-676, 2004.

[7] B. Dong and W. Jiang, "On the decay of higher order derivatives of solutions to Ladyzhenskaya model for incompressible viscous flows," Science in China A: Mathematics, vol. 51, no. 5, pp. 925934, 2008.

[8] M. E. Schonbek, " $L^{2}$ decay for weak solutions of the NavierStokes equations," Archive for Rational Mechanics and Analysis, vol. 88, no. 3, pp. 209-222, 1985.

[9] M. F. Wheeler, "A priori $L^{2}$ error estimates for Galerkin approximations to parabolic partial differential equations," SIAM Journal on Numerical Analysis, vol. 10, pp. 723-759, 1973.

[10] D. Gilbarg and N. Trudinger, Elliptic Partial Differential Equations of Second Order, Springer, New York, NY, USA, 3rd edition, 2001.

[11] D. Henry, Geometric Theory of Semilinear Parabolic Equations, Springe, New York, NY, USA, 1981.

[12] M. Dai, J. Qing, and M. Schonbek, "Asymptotic behavior of solutions to liquid crystal systems in $\mathbb{R}^{3}$," Communications in Partial Differential Equations, vol. 37, no. 12, pp. 2138-2164, 2012.

[13] C. Jin, J. Yin, and C. Wang, "Large time behaviour of solutions for the heat equation with spatio-temporal delay," Nonlinearity, vol. 21, pp. 823-840, 2008.

[14] B. Dong and Z. Chen, "Asymptotic profiles of solutions to the $2 \mathrm{D}$ viscous incompressible micropolar fluid flows," Discrete and Continuous Dynamical Systems A, vol. 23, no. 3, pp. 765-784, 2009.

[15] M. E. Schonbek, "Decay of solutions to parabolic conservation laws," Communications in Partial Differential Equations, vol. 5, no. 5, pp. 449-473, 1980.

[16] B. Dong, Y. Jia, and Z. Chen, "Pressure regularity criteria of the three-dimensional micropolar fluid flows," Mathematical Methods in the Applied Sciences, vol. 34, no. 5, pp. 595-606, 2011.

[17] B. Dong and Z. Chen, "Asymptotic stability of the critical and super-critical dissipative quasi-geostrophic equation," Nonlinearity, vol. 19, no. 12, pp. 2919-2928, 2006.

[18] R. Ricci, "Large time behavior of the solution of the heat equation with nonlinear strong absorption," Journal of Differential Equations, vol. 79, no. 1, pp. 1-13, 1989.

[19] P. Secchi, " $L^{2}$ stability for weak solutions of the Navier-Stokes equations in $\mathbb{R}^{3}$," Indiana University Mathematics Journal, vol. 36, no. 3, pp. 685-691, 1987.

[20] B. Dong and J. Song, "Global regularity and asymptotic behavior of modified Navier-Stokes equations with fractional dissipation," Discrete and Continuous Dynamical Systems, vol. 32, no. 1, pp. 57-79, 2012. 


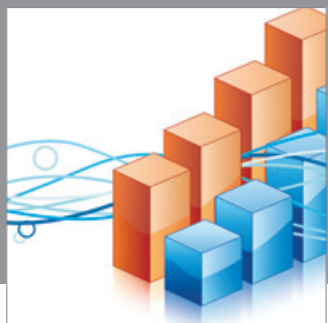

Advances in

Operations Research

mansans

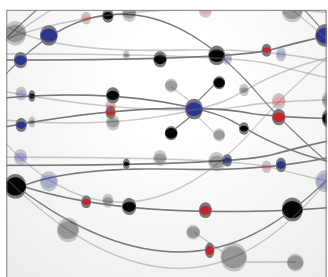

The Scientific World Journal
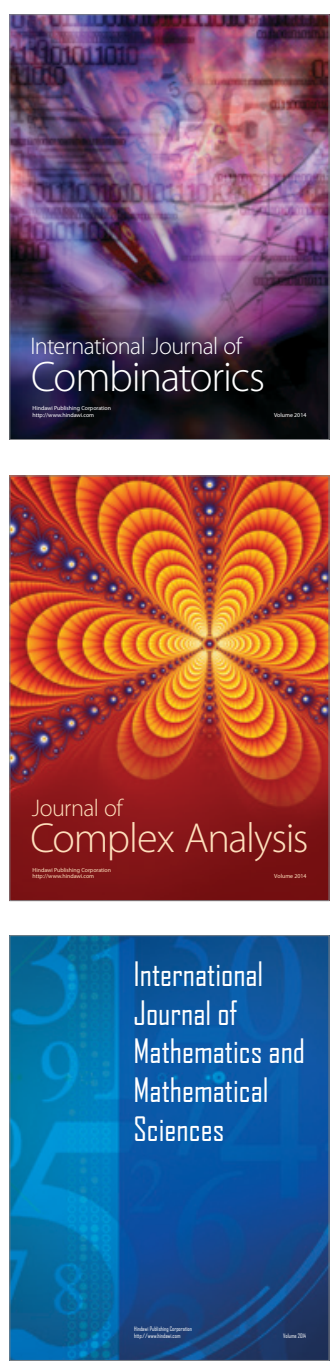
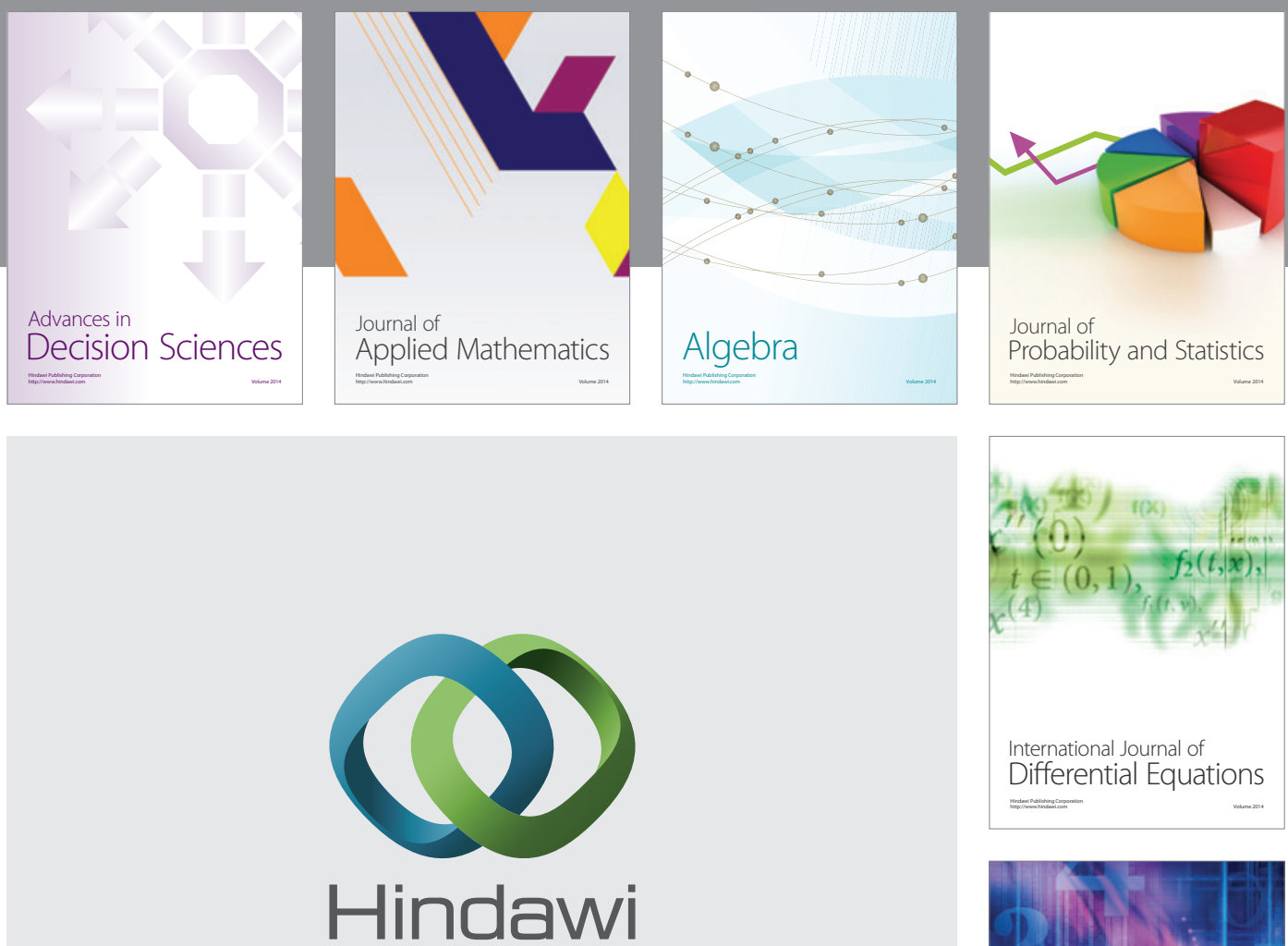

Submit your manuscripts at http://www.hindawi.com
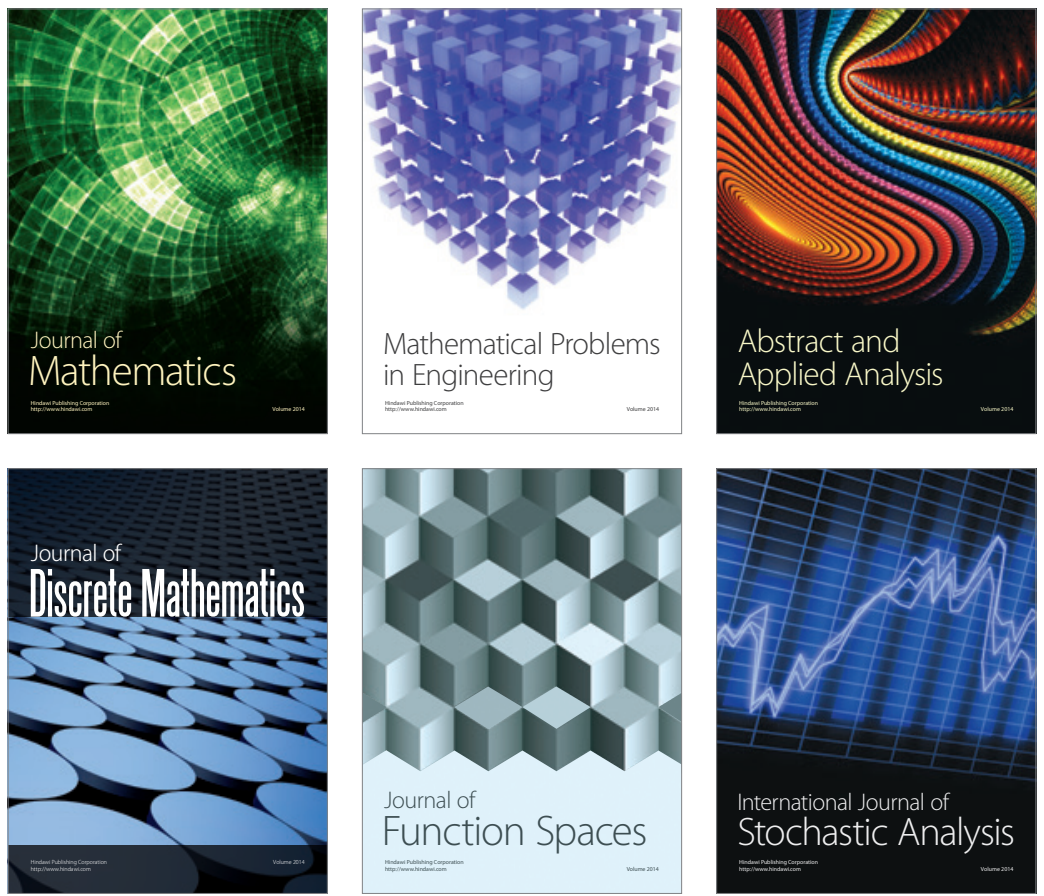

Journal of

Function Spaces

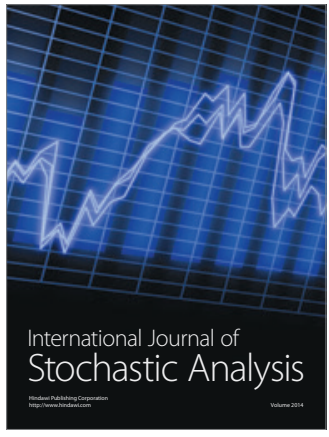

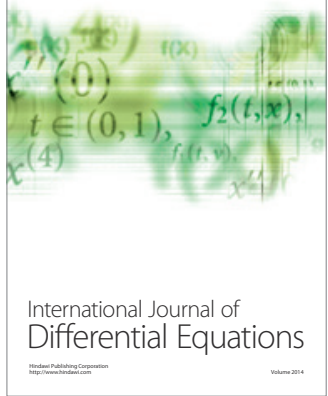
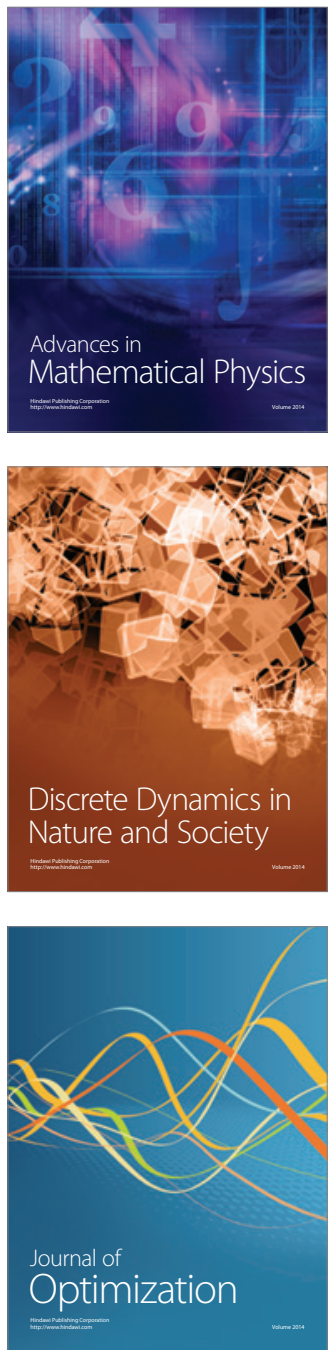